\title{
Generalized Hofstadter model on a cubic optical lattice: From nodal bands to the three-dimensional quantum Hall effect
}

\author{
Dan-Wei Zhang, ${ }^{1, *}$ Rui-Bin Liu, ${ }^{2}$ and Shi-Liang Zhu ${ }^{3,1,4, \dagger}$ \\ ${ }^{1}$ Guangdong Provincial Key Laboratory of Quantum Engineering and Quantum Materials, \\ SPTE, South China Normal University, Guangzhou 510006, China \\ ${ }^{2}$ Department of Physics and Center of Theoretical and Computational Physics, The University of Hong Kong, \\ Pokfulam Road, Hong Kong, China \\ ${ }^{3}$ National Laboratory of Solid State Microstructures and School of Physics, Nanjing University, Nanjing 210093, China \\ ${ }^{4}$ Synergetic Innovation Center of Quantum Information and Quantum Physics, University of Science and Technology of China, \\ Hefei, Anhui 230026, China
}

(Received 19 February 2017; published 17 April 2017)

\begin{abstract}
We propose that a tunable generalized three-dimensional Hofstadter Hamiltonian can be realized by engineering the Raman-assisted hopping of ultracold atoms in a cubic optical lattice. The Hamiltonian describes a periodic lattice system under artificial magnetic fluxes in three dimensions. For certain hopping configurations, the bulk bands can have Weyl points and nodal loops, respectively, allowing the study of both the two nodal semimetal states within this system. Furthermore, we illustrate that with proper rational fluxes and hopping parameters, the system can exhibit the three-dimensional quantum Hall effect when the Fermi level lies in the band gaps, which is topologically characterized by one or two nonzero Chern numbers. Our proposed optical-lattice system provides a promising platform for exploring various exotic topological phases in three dimensions.
\end{abstract}

DOI: 10.1103/PhysRevA.95.043619

\section{INTRODUCTION}

Topological states of matter have attracted great interest since the discovery of the quantum Hall effect with intrinsic topological invariants in two-dimensional electron systems $[1,2]$. Early work was devoted to constructing theoretical models in this context $[3,4]$, including the celebrated Haldane model [5] and Hofstadter model [6]. Following the lines of the two-dimensional work [3], it has been shown that if there is an energy gap in a three-dimensional periodic lattice, then the integer quantum Hall effect can result when the Fermi energy lies inside the gap [7-10]. In the three-dimensional quantum Hall effect, the Hall conductance in each crystal plane can have a quantized Hall value, which is a topological invariant - the first Chern number of a U(1) principal fiber bundle of a torus spanned by the two quasimomenta for the crystal plane. However, obtaining the energy spectrum with band gaps for the emergence of quantized Hall conductivities in three-dimensional periodic lattices is a nontrivial task since a motion along the third direction may wash out the gaps of the perpendicular two-dimensional plane [8-10]. In recent years, significant advances have been made in the study of band topology of insulating and semimetallic materials, such as topological insulators $[11,12]$ and topological nodal semimetals with Weyl points or nodal loops (bands crossing along closed lines instead of isolated points) in three-dimensional momentum space [13-17]. Notably, a three-dimensional Dirac point described by the four-component Dirac equation is composed by two Weyl points with opposite chirality, and they can be separated in momentum space by breaking either timereversal symmetry or inversion symmetry. The low-energy excitations (Weyl quasiparticles) near the Weyl points are

\footnotetext{
*zdanwei@126.com

†slzhu@nju.edu.cn
}

described by the Weyl equation, which is a massless twocomponent Dirac equation in the chiral representation. These exotic nodal semimetal states are still rare in real materials or artificial systems $[18,19]$, and the three-dimensional quantum Hall effect has been predicted or observed only in systems with extreme anisotropy or unconventional toroidal magnetic fields [10,20-23].

On the other hand, ultracold atomic gases in optical lattices provide unparalleled controllability and new avenues to simulate various quantum states in condensed matter physics $[24,25]$. Recent experimental advances in engineering artificial gauge field and spin-orbit coupling for neutral atoms [26-34] have pushed this system to the forefront for exploring topological phases [35-47]. For instance, the Zak phase in topologically nontrivial Bloch bands realized in one-dimensional optical superlattices has been measured [48]. The Haldane model and the Hofstadter model have been realized with synthetic magnetic fluxes in two-dimensional optical lattices [49-53], and the Chern number characterizing the topological bands has been measured [53]. The technique of Bloch band tomography in optical lattices has been proposed and experimentally demonstrated [54-57]. In addition, it has been separately proposed to simulate Weyl points and nodal loops by engineering atomic Hofstadter bands or spin-orbit coupling [58-65]. It thus would be of great value to set an experimentally feasible stage for quantum simulation of both the two exotic nodal semimetal states and the rarely explored three-dimensional quantum Hall effect within an optical-lattice system.

In this paper, we propose an experimental scheme to realize a tunable generalized three-dimensional Hofstadter Hamiltonian with ultracold atoms in a cubic optical lattice based on the Raman-assisted hopping method. The original Hofstadter model describes charged particles moving in a two-dimensional lattice penetrated by a uniform magnetic flux, which develop the quantized energy spectrum and Hall conductivity. The synthetically generated Hamiltonian in our 
proposal describes a three-dimensional periodic lattice system under three tunable effective magnetic fluxes, which can thus be viewed as a generalized Hofstadter Hamiltonian and be used to simulate various topological phases. We first show that the Weyl points and nodal loops can respectively emerge in the bulk bands of this system for certain hopping configurations, allowing the study of both the two topological nodal semimetal states within this system. Furthermore, by numerically elaborating the energy spectra and the topological invariants, we illustrate that for proper rational fluxes and hopping parameters, the system can exhibit the three-dimensional quantum Hall effect when the Fermi level lies in the band gaps, which is topologically characterized by one or two nonzero Chern numbers. Our proposed optical-lattice system provides a powerful platform for exploring exotic topological semimetals and insulators in three dimensions that are rare in real materials.

The paper is organized as follows. Section II introduces the experimental scheme to realize the tunable three-dimensional Hofstadter Hamiltonian in a cubic optical lattice. In Sec. III, we show that bulk bands of this system can, respectively, have Weyl points and nodal loops for certain hopping configurations. In Sec. IV, we illustrate the emergence of the three-dimensional quantum Hall effect in the system with numerical calculation of the energy spectra and the topological invariants. Finally, a short conclusion is given in Sec. V.

\section{SYSTEM AND MODEL}

We consider an ultracold degenerate gas of fermionic atoms in a cubic optical lattice tilted along the $y$ and $z$ axes with $a$ being the lattice spacing, as shown in Fig. 1(a). The atoms are prepared in a hyperfine state of the ground-state manifold, and the tilt potentials with linear energy shift per lattice site $\Delta_{S}$
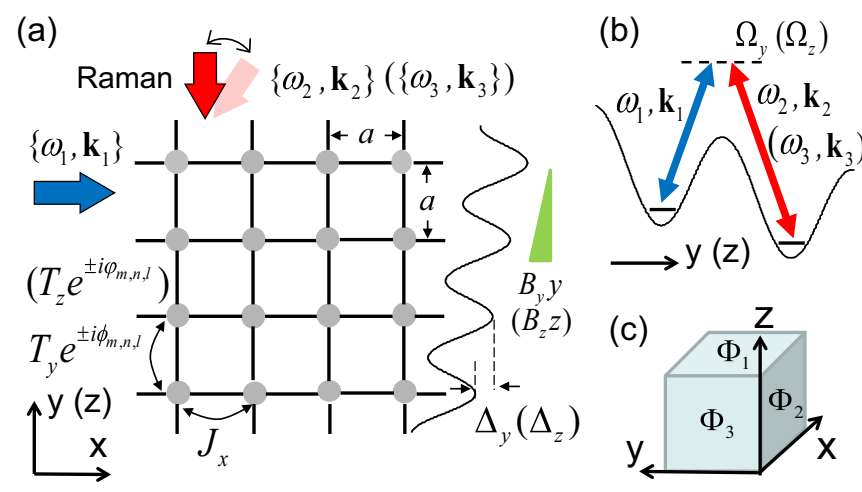

FIG. 1. Proposed realization of a generalized Hofstadter model in three dimensions. (a) The optical lattice and hopping configuration. The atomic hopping along the $x$ axis is $J_{x}$. Along the $s(s=y, z)$ axis, the tilted lattice with large tilt potentials $\Delta_{s}$ can be created by the gravity or real magnetic field gradients $B_{s} s$. The natural hopping along the $s$ axis is suppressed and can then be restored by using three far-detuned Raman lasers denoted by $\left\{\omega_{j}, \mathbf{k}_{j}\right\}(j=1,2,3)$, which give rise to complex hopping amplitudes $T_{y} e^{ \pm i \phi_{m, n, l}}$ and $T_{z} e^{ \pm i \varphi_{m, n, l}}$ with site indices $(m, n, l)$. (b) Laser-assisted tunneling between nearestneighboring sites along the $s$ axis with the frequency differences $\omega_{2}-\omega_{1}=\Delta_{y} / \hbar$ and $\omega_{3}-\omega_{1}=\Delta_{z} / \hbar$ and the effective two-photon Rabi frequency $\Omega_{s}$. (c) The effective magnetic fluxes $\left\{\Phi_{1}, \Phi_{2}, \Phi_{3}\right\}$ in the three elementary plaquettes in the $\{x y, x z, y z\}$ planes, respectively. $(s=y, z)$ can be generated by the gravity or real magnetic field gradients $B_{s} s$, respectively. For the cases $\Delta_{s} \gg J_{s}$ we considered, where $J_{s}$ denotes the bare hopping amplitude along the $s$ axis, the atomic hopping between neighboring sites in these two directions is then suppressed. To restore and engineer the hopping terms with tunable effective Peierls phases, we can use the Raman-assisted tunneling technique [66,67], which has been experimentally demonstrated to realize the original Hofstadter model in two-dimensional optical lattices [50-53]. This method has also been adopted to simulate Weyl points [59]. Now we extend the schemes to realize a generalized three-dimensional Hofstadter Hamiltonian with fully tunable hopping parameters.

In order to fully and independently engineer the atomic hopping along the $y$ and $z$ axes in this optical-lattice system, one can use three far-detuned Raman beams denoted by their frequencies and wave vectors $\left\{\omega_{j}, \mathbf{k}_{j}\right\}(j=1,2,3)$, as shown in Fig. 1(b). Here we choose the frequency differences $\omega_{2}-$ $\omega_{1}=\Delta_{y} / \hbar$ and $\omega_{3}-\omega_{1}=\Delta_{z} / \hbar$ for the resonant tunneling condition, and assume the effective two-photon Rabi frequencies $\Omega_{s}$. Note that we consider $\Delta_{y} \neq \Delta_{z}$, which is required for resonant tunneling along different directions. In addition, the momentum transfers $\mathbf{Q}=\mathbf{k}_{\mathbf{1}}-\mathbf{k}_{\mathbf{2}} \equiv\left(Q_{x}, Q_{y}, Q_{z}\right)$ and $\mathbf{P}=\mathbf{k}_{\mathbf{1}}-\mathbf{k}_{\mathbf{3}} \equiv\left(P_{x}, P_{y}, P_{z}\right)$ can be independently tunable, for instance, through independently adjusting the angles of the second and third Raman lasers with the first Raman laser being fixed, as shown in Fig. 1(a). Therefore, the Raman lasers induce atomic hopping along the $y$ and $z$ axis with tunable spatially dependent phases $\phi_{m, n, l}=\mathbf{Q} \cdot \mathbf{R}=m \phi_{x}+n \phi_{y}+l \phi_{z}$ and $\varphi_{m, n, l}=\mathbf{P} \cdot \mathbf{R}=m \varphi_{x}+n \varphi_{y}+l \varphi_{z}$, respectively, where $\mathbf{R}=(m a, n a, l a)$ denotes the position vector for the lattice site $(m, n, l), \phi_{x, y, z}=a Q_{x, y, z}$ and $\varphi_{x, y, z}=a P_{x, y, z}$. In the highfrequency limit $\omega_{j} \gg J_{s} / \hbar$ [68], time averaging over rapidly oscillating Raman beams yields an effective time-independent Hamiltonian [51-53]. As a result, the tilts disappear in the dressed atom picture for resonant tunneling. In this case, the effective time-independent Hamiltonian for the threedimensional lattice system takes the tight-binding form:

$$
\begin{aligned}
\hat{H}= & -\sum_{m, n, l} J_{x} \hat{a}_{m+1, n, l}^{\dagger} \hat{a}_{m, n, l}+T_{y} e^{i \phi_{m, n, l}} \hat{a}_{m, n+1, l}^{\dagger} \hat{a}_{m, n, l} \\
& +T_{z} e^{i \varphi_{m, n, l}} \hat{a}_{m, n, l+1}^{\dagger} \hat{a}_{m, n, l}+\text { H.c. },
\end{aligned}
$$

where $\hat{a}_{m, n, l}^{\dagger}\left(\hat{a}_{m, n, l}\right)$ is the creation (annihilation) operator of fermions at the lattice site $(m, n, l), J_{x}$ is the natural hopping along the $x$ axis, and $T_{y} e^{i \phi_{m, n}}\left(T_{z} e^{i \varphi_{m, l}}\right)$ denotes the Raman-induced hopping along the $y(z)$ axis with the spatially varying phase $\phi_{m, n}\left(\varphi_{m, l}\right)$ imprinted by the Raman lasers. The engineered hopping strengths $T_{s}=\Omega_{s} \lambda_{s}$ can also be tuned through changing the intensities of the Raman lasers, where $\lambda_{s}$ denotes the overlap integral of Wannier-Stark functions between neighbor sites along the $s$ axis [51-53].

The three-dimensional Bravais lattice here is spanned by the primitive vectors $\mathbf{a}_{x}, \mathbf{a}_{y}$, and $\mathbf{a}_{z}$. One can introduce three effective magnetic fluxes $\left\{\Phi_{1}, \Phi_{2}, \Phi_{3}\right\}$ through the three elementary plaquettes in the $\{x y, x z, y z\}$ planes with the area $S=a^{2}$, as shown in Fig. 1(c). The effective fluxes, in units of the magnetic flux quantum, are determined by the phases picked up anticlockwise around the plaquettes from 
Hamiltonian (1), which are obtained as $\Phi_{1}=\frac{1}{2 \pi}\left(0+\phi_{m+1, n, l}+\right.$ $\left.0-\phi_{m, n, l}\right)=\frac{\phi_{x}}{2 \pi}, \quad \Phi_{2}=\frac{1}{2 \pi}\left(0+\varphi_{m+1, n, l}+0-\varphi_{m, n, l}\right)=\frac{\varphi_{x}}{2 \pi}$, $\Phi_{3}=\frac{1}{2 \pi}\left(\varphi_{m, n, l}+\phi_{m, n, l+1}-\varphi_{m, n+1, l}-\phi_{m, n, l}\right)=\frac{\phi_{z}-\varphi_{y}}{2 \pi}$. The three effective magnetic fluxes can thus be independently tuned from zero to positive or negative 1 , and we here focus on the positive-flux cases since the opposite flux configurations reproduce the same results.

If one of the atomic hopping strengths along three directions in Hamiltonian (1) becomes zero, the system reduces to a stack of decoupled two-dimensional Hofstadter systems with cold atoms [51-53]. Thus, in general cases without vanishing hopping, the system corresponds to three copies of the original Hofstadter model defined in connected planes. In this sense, Hamiltonian (1) can be regarded as a generalized threedimensional Hofstadter Hamiltonian, which describes an effectively charged particle hopping on a tight-binding cubic lattice in the presence of tunable uniform magnetic fluxes [7-10].

\section{WEYL POINTS AND NODAL LOOPS}

The hopping and flux parameters in Hamiltonian (1) are tunable through the laser configuration, enabling the opticallattice system for simulating various topological states. In this section, we show that for certain hopping configurations, the bulk bands of the system can respectively have Weyl points and nodal loops, which allow one to study both the topological Weyl and nodal-loop semimetals within this cold atom system.

We first choose the hopping parameters $\phi_{x, y}=\varphi_{x, y}=$ $\phi_{z} / 2=\varphi_{z} / 2=1 / 2$. In this configuration, the hopping phase along the $s$ axis (both the $y$ and $z$ axes) is $\pi(0)$, for the site indices $m+n$ odd (even). Thus the lattice system has two sublattices $A$ and $B$, which correspond to $m+n$ odd and even, respectively. In this case, Hamiltonian (1) can be rewritten as

$$
\begin{aligned}
\hat{H}_{1}= & -\sum_{A}\left(J_{x} \hat{b}_{A+\hat{x}}^{\dagger} \hat{a}_{A}-T_{y} \hat{b}_{A+\hat{y}}^{\dagger} \hat{a}_{A}-T_{z} \hat{a}_{A+\hat{z}}^{\dagger} \hat{a}_{A}\right) \\
& -\sum_{B}\left(J_{x} \hat{a}_{B+\hat{x}}^{\dagger} \hat{b}_{B}+T_{y} \hat{a}_{B+\hat{y}}^{\dagger} \hat{b}_{B}+T_{z} \hat{b}_{B+\hat{z}}^{\dagger} \hat{b}_{B}\right)+\text { H.c. }
\end{aligned}
$$

where $\hat{a}_{A}^{\dagger}=\hat{a}_{B+\hat{\eta}}^{\dagger}$ and $\hat{a}_{A}=\hat{a}_{B+\hat{\eta}}\left(\hat{b}_{B}^{\dagger}=\hat{b}_{A+\hat{\eta}}^{\dagger}\right.$ and $\left.\hat{b}_{B}=\hat{b}_{A+\hat{\eta}}\right)$ are the creation and annihilation operators at $A(B)$ sublattices with $\eta=x, y, z$ denoting hopping along the $\eta$ direction. The two sublattices form pseudospin indices, and Hamiltonian (2) can then be transformed as $\hat{H}_{1}=\sum_{\mathbf{k}} \hat{\Psi}_{\mathbf{k}}^{\dagger} \mathcal{H}_{1}(\mathbf{k}) \hat{\Psi}_{\mathbf{k}}$ with $\hat{\Psi}_{\mathbf{k}}^{\dagger}=$ $\left(\hat{a}_{\mathbf{k}}^{\dagger}, \hat{b}_{\mathbf{k}}^{\dagger}\right)$ and the Bloch Hamiltonian

$$
\begin{aligned}
\mathcal{H}_{1}(\mathbf{k})= & -2\left[J_{x} \cos \left(k_{x} a\right) \sigma_{x}\right. \\
& \left.+T_{y} \sin \left(k_{y} a\right) \sigma_{y}+T_{z} \cos \left(k_{z} a\right) \sigma_{z}\right],
\end{aligned}
$$

where $\mathbf{k}=\left(k_{x}, k_{y}, k_{z}\right)$ and $\sigma_{x, y, z}$ are the Pauli matrices acting on the sublattices. The two bulk bands $E_{1, \pm}(\mathbf{k})=$ $\pm 2 \sqrt{J_{x}^{2} \cos ^{2}\left(k_{x} a\right)+T_{y}^{2} \sin ^{2}\left(k_{y} a\right)+T_{z}^{2} \cos ^{2}\left(k_{z} a\right)}$ have four Weyl points located at $\mathbf{k}_{W}=( \pm \pi / 2 a, 0, \pm \pi / 2 a)$ in the first Brillouin zone. Figure 2(b) depicts the spectrum $E_{1,+}(\mathbf{k})$ with the four Weyl points. In the vicinity of $\mathbf{k}_{W}$ with $\mathbf{q}=\mathbf{k}-\mathbf{k}_{W}$, one can obtain an effective Weyl Hamiltonian, $\mathcal{H}_{W}(\mathbf{q})=$ $v_{x} q_{x} \sigma_{x}+v_{y} q_{y} \sigma_{y}+v_{z} q_{z} \sigma_{z}$, where $v_{x}= \pm 2 J_{x} a, v_{y}=-2 T_{y} a$, and $v_{z}= \pm 2 T_{z} a$ for the four points. The topological nature of (a)

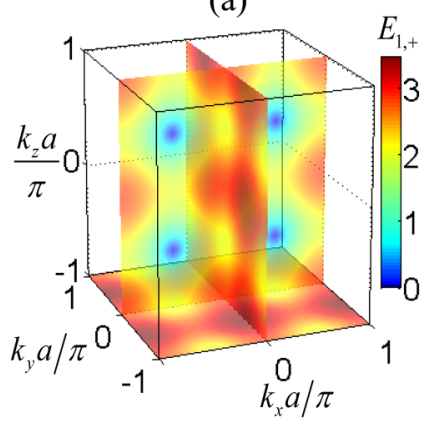

(b)

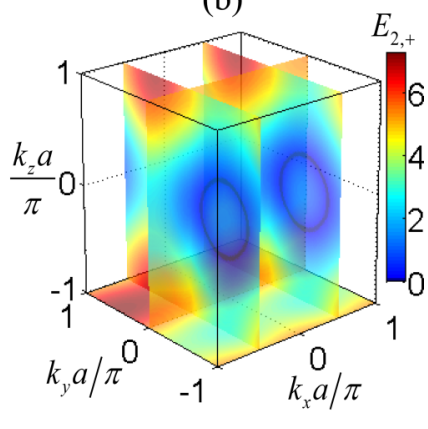

FIG. 2. Simulated Weyl points and nodal loops. The color-coded three-dimensional bulk spectra: (a) $E_{1,+}$ (k) with four Weyl points and (b) $E_{2,+}(\mathbf{k})$ with two nodal loops in the $k_{x}= \pm \pi / 2 a$ planes. The parameters are $T_{y}=T_{z}=\epsilon / 3=J_{x}=1$.

the Weyl points is characterized by their positive or negative chirality defined as $\kappa=\operatorname{sgn}\left(v_{x} v_{y} v_{z}\right)$. Note that the effective Weyl Hamiltonian simulated here recovers the one proposed in Ref. [59] by a pseudospin rotation.

For another hopping configuration given by $\phi_{x}=\varphi_{x}=$ $\phi_{y, z} / 2=\varphi_{y, z} / 2=1 / 2$ (here $\phi_{z}$ and $\varphi_{y}$ can alternatively be $\left.\phi_{z}=\varphi_{y}=0\right)$, the nodal loops can be exhibited in the bulk bands of the system. In this case, the atomic hopping along the $y$ axis and the two sublattices $(A$ and $B)$ are both staggered along the $x$ axis. In addition, one can add a tunable energy offset between the two sublattices through a small one-photon detuning $\epsilon=\Delta_{y}-\hbar\left(\omega_{2}-\omega_{1}\right)=\Delta_{z}-\hbar\left(\omega_{3}-\omega_{1}\right)$ in the Raman coupling. Under this circumstance, Hamiltonian (1) turns to the form

$$
\begin{aligned}
\hat{H}_{2}= & -\sum_{A}\left(J_{x} \hat{b}_{A+\hat{x}}^{\dagger} \hat{a}_{A}-T_{y} \hat{a}_{A+\hat{y}}^{\dagger} \hat{a}_{A}-T_{z} \hat{a}_{A+\hat{z}}^{\dagger} \hat{a}_{A}\right) \\
& -\sum_{B}\left(J_{x} \hat{a}_{B+\hat{x}}^{\dagger} \hat{b}_{B}+T_{y} \hat{b}_{B+\hat{y}}^{\dagger} \hat{b}_{B}+T_{z} \hat{b}_{B+\hat{z}}^{\dagger} \hat{b}_{B}\right) \\
& +\epsilon \sum_{A, B}\left(\hat{a}_{A}^{\dagger} \hat{a}_{A}-\hat{b}_{B}^{\dagger} \hat{b}_{B}\right)+\text { H.c. }
\end{aligned}
$$

This gives rise to the Bloch Hamiltonian

$$
\mathcal{H}_{2}(\mathbf{k})=-2 J_{x} \cos \left(k_{x} a\right) \sigma_{x}-g\left(k_{y}, k_{z}\right) \sigma_{z},
$$

where $g\left(k_{y}, k_{z}\right)=2 T_{y} \sin \left(k_{y} a\right)+2 T_{z} \cos \left(k_{z} a\right)-\epsilon$. The bulk bands $E_{2, \pm}(\mathbf{k})= \pm \sqrt{4 J_{x}^{2} \cos ^{2}\left(k_{x} a\right)+\left[g\left(k_{y}, k_{z}\right)\right]^{2}}$ have twofold degenerate points located at $k_{x}= \pm \pi / 2 a$ planes with $k_{y}$ and $k_{z}$ satisfying the condition $g\left(k_{y}, k_{z}\right)=0$. The solutions of the condition function can give rise to the nodal loops for proper parameter $\epsilon$. Figure 2(b) depicts an example of the bulk spectrum $E_{2,+}(\mathbf{k})$ with two nodal loops in the $k_{x}= \pm \pi / 2 a$ planes, respectively.

With fermionic atoms in the optical lattice system, one can explore the two exotic nodal states, Weyl and nodal-loop semimetals. In realistic experiments, the simulated nodal points and loops in the three-dimensional Brillouin zone can be verified by probing the momentum distribution of the atomic transfer fraction in the excited band after BlochZener oscillations through the time-of-flight measurements $[60,63,69,70]$. 


\section{THE THREE-DIMENSIONAL QUANTUM HALL EFFECT}

In this section, we will demonstrate that the proposed cold atom system can be used to realize the exotic integer quantum Hall effect in three dimensions. For simplicity but without loss of generality, we focus on the cases with zero magnetic flux in the $y z$ plane $\Phi_{3}=0$, which can be achieved by choosing the parameters $\phi_{z}=\varphi_{y}$. We consider that the effective magnetic fluxes $\Phi_{1}$ and $\Phi_{2}$ are rational, i.e., $\Phi_{1}=p_{1} / q_{1}$ and $\Phi_{2}=$ $p_{2} / q_{2}$, with mutually prime integers $p_{1,2}$ and $q_{1,2}$. In addition, a gauge is chosen in which the hopping phases $\phi_{m, n, l}=2 \pi \Phi_{1} m$ and $\varphi_{m, n, l}=2 \pi \Phi_{2} m$ in Hamiltonian (1), which corresponds to the Landau gauge in the continuum case and can be achieved with the parameters, e.g., $\phi_{y}=\varphi_{z}=2 \pi$ and $\phi_{z}=\varphi_{y}=0$. The nonvanishing momentum transfers in the two tilt directions here (i.e., $\phi_{y}=\varphi_{z}=2 \pi$ ) are necessary for restoring the resonant tunneling $[48,51,53]$. For the neutral atomic system within this choice of gauge, the effective vector potential is given by $\mathbf{A}=\left(0, B_{z} x,-B_{y} x\right)$, with $\mathbf{B}=\left(0, B_{y}, B_{z}\right)$ denoting an effective magnetic field in the $y z$ plane and $x=m a$.

\section{A. The energy spectra}

An essential point in the quantum Hall effect is that the Fermi level lies in one of the gaps in the bulk spectrum, such as the butterfly energy spectrum in the two-dimensional Hofstadter system. So we first study the energy spectrum in this tunable three-dimensional Hofstadter optical lattice system. Considering $y=n a$ and $z=l a$ as the periodic coordinates on the system, Hamiltonian (1) can be block diagonalized as $\hat{H}=$ $\bigoplus \hat{H}_{x}\left(k_{y}, k_{z}\right)$, where $k_{y}$ and $k_{z}$ are the quasimomenta along the periodic directions and the decoupled block Hamiltonian under the above conditions takes the following form:

$$
\hat{H}_{x}\left(k_{y}, k_{z}\right)=-\sum_{m}\left(J_{x} \hat{a}_{m+1}^{\dagger} \hat{a}_{m}+\text { H.c. }\right)-\sum_{m} V_{m} \hat{a}_{m}^{\dagger} \hat{a}_{m},
$$

where $V_{m}=2 T_{y} \cos \left(2 \pi \Phi_{1} m+k_{y} a\right)+2 T_{z} \cos \left(2 \pi \Phi_{2} m+k_{z} a\right)$. The corresponding single-particle wave function $\Psi_{m n l}$ is written as $\Psi_{m n l}=e^{i k_{y} y+i k_{z} z} \psi_{m}$, and then the Schrödinger equation $\hat{H}_{x}\left(k_{y}, k_{z}\right) \Psi_{m n l}=E \Psi_{m n l}$ reduces to a generalized Harper equation [71] with the parameters $k_{y}$ and $k_{z}$ as

$$
-J_{x}\left(\psi_{m-1}+\psi_{m+1}\right)-V_{m} \psi_{m}=E \psi_{m} .
$$

This reduced one-dimensional tight-binding system with two commensurabilities $\Phi_{1}$ and $\Phi_{2}$ has a period of the least common multiple of integers $q_{1}$ and $q_{2}$ denoted by $\tilde{q}=\left[q_{1}, q_{2}\right]$. Under the periodic boundary condition along the $x$ axis, the wave function $\psi_{m}$ satisfies $\psi_{m}=e^{i k_{x} x} u_{m}(\mathbf{k})$ with $u_{m}(\mathbf{k})=u_{m+\tilde{q}}(\mathbf{k})$. Therefore in a general case, the spectrum of the three-dimensional system in the presence of the effective magnetic fluxes consists of $\tilde{q}$ energy bands and each band has a reduced (magnetic) Brillouin zone: $-\pi / \tilde{q} a \leqslant k_{x} \leqslant$ $\pi / \tilde{q} a,-\pi / a \leqslant k_{y} \leqslant \pi / a,-\pi / a \leqslant k_{z} \leqslant \pi / a$. In terms of the reduced Bloch wave function $u_{m}(\mathbf{k})$, Eq. (7) becomes

$$
-J_{x}\left(e^{i k_{x}} u_{m-1}+e^{-i k_{x}} u_{m+1}\right)-V_{m} u_{m}=E(\mathbf{k}) u_{m} .
$$

Since $u_{m}(\mathbf{k})=u_{m+\tilde{q}}(\mathbf{k})$, the problem of solving the generalized Harper equation (8) reduces to solving the eigenvalue equation, $M \Upsilon=E \Upsilon$, where $\Upsilon=\left(u_{1}, \ldots, u_{\tilde{q}}\right)$ is the wave function for the $\tilde{q}$ bands and $M$ is the $\tilde{q} \times \tilde{q}$ matrix.

In order to confirm the above analysis, we numerically calculate the energy spectra of the system from exact diagonalization of the tight-binding Hamiltonian (6) under the periodic boundary condition. We first consider the evolution of the spectra with the increasing of the hopping strength $T_{z}$ for fixed typical magnetic fluxes and $T_{y}$ (and $J_{x}=1$ as the energy unit). As shown in Fig. 3, one can find that the overlapping of the $\tilde{q}$ bands depend on the hopping strength $T_{z}$. Figures 3(a) and 3(b) show the structures of these bands for the fluxes $\Phi_{2}=2 \Phi_{1}=2 / 5$ with $\tilde{q}=5$ and $\Phi_{2}=2 \Phi_{1}=2 / 7$ with $\tilde{q}=7$, respectively. In these cases, the largest number of gaps $N_{g}=\tilde{q}-1$ is available for the $\tilde{q}$ bands that are totally nonoverlapping, which can be achieved when the
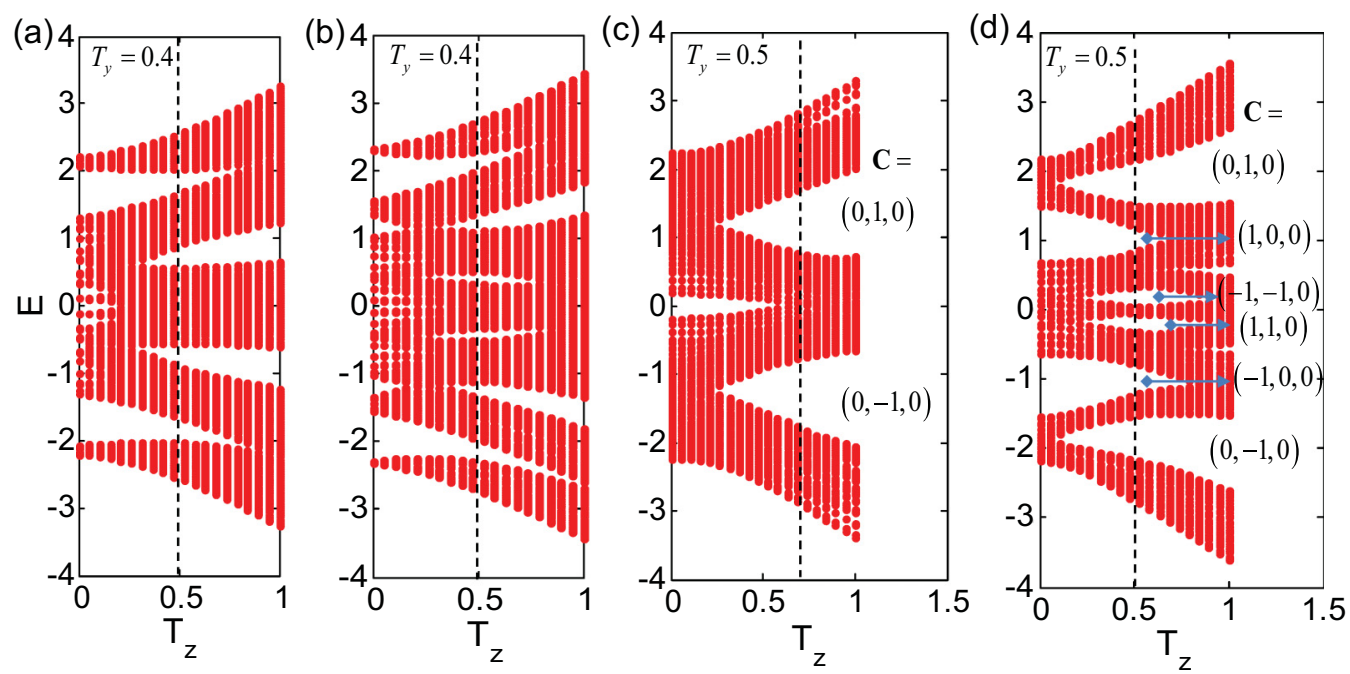

FIG. 3. The energy spectra $E$ as a function of the hopping strength $T_{z}$ for typical flux and hopping parameters: (a) $\Phi_{1}=1 / 5, \Phi_{2}=2 / 5$, and $T_{y}=0.4$; (b) $\Phi_{1}=1 / 7, \Phi_{2}=2 / 7$, and $T_{y}=0.4$; (c) $\Phi_{1}=1 / 2, \Phi_{2}=1 / 3$, and $T_{y}=0.5$; (d) $\Phi_{1}=1 / 3, \Phi_{2}=1 / 5$, and $T_{y}=0.5$. In (c) with $T_{z}=0.7$ and in (d) with $T_{z}=0.5$ shown as the dashed lines, the Chern numbers $\mathbf{C}=\left(C_{x y}, C_{x z}, C_{y z}\right)$ when the Fermi level lies in each energy gap are plotted. The energy unit is set as $J_{x}=1$. 

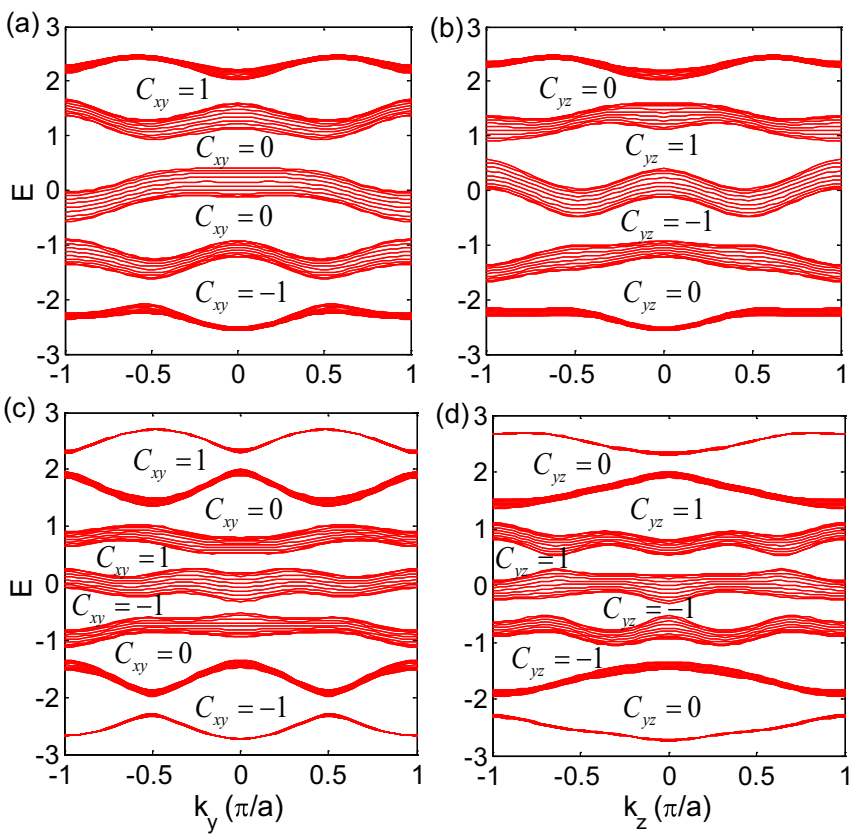

FIG. 4. The energy spectra $E\left(k_{y}\right)$ and $E\left(k_{z}\right)$ and the corresponding Chern numbers $C_{x y}$ and $C_{x z}$. (a) and (b) $\Phi_{1}=1 / 5$ and $\Phi_{2}=2 / 5$; (c) and (d) $\Phi_{1}=1 / 7$ and $\Phi_{2}=2 / 7$. We choose $k_{z}=0$ in (a) and (c), and $k_{y}=0$ in (b) and (d). The hopping strengths are $T_{y}=0.4$ and $T_{z}=0.5$ in (a)-(d), as the dashed lines shown in Figs. 3(a) and 3(b). The energy unit is set as $J_{x}=1$.

hopping strength $T_{z}$ is close to $T_{y}$, such as the dashed lines shown in Figs. 3(a) and 3(b). Figures 3(c) and 3(d) show the bands for the fluxes $\left\{\Phi_{1}=1 / 2, \Phi_{2}=1 / 3\right\}$ with $\tilde{q}=6$ and $\left\{\Phi_{1}=1 / 3, \Phi_{2}=1 / 5\right\}$ with $\tilde{q}=15$, respectively. In these cases, the largest number of gaps always are $N_{g}<\tilde{q}-1$ for different $T_{z}$ because some of the $\tilde{q}$ bands overlap with each other, such as $N_{g}=3$ and $N_{g}=6$ for the dashed lines shown in Figs. 3(c) and 3(d), respectively. For the cases in Figs. 3(a) and 3(b) with the bands being totally nonoverlapping, in Fig. 4, we plot the energy spectra for the reduced two-dimensional subsystems $E\left(k_{y}\right)$ and $E\left(k_{z}\right)$ for fixed $k_{z}=0$ and $k_{y}=0$, respectively. The energy spectra $E\left(k_{y}\right)$ and $E\left(k_{z}\right)$ there reproduce the ones for the two-dimensional Hofstadter systems with $\tilde{q}=5$ bands in Figs. 4(a) and 4(b) and with $\tilde{q}=7$ bands in Figs. 4(c) and 4(d).

Due to the fact that the bands overlap in energy or touch each other, there is usually no analogous butterfly spectrum with fractal energy gaps in this three-dimensional generalized Hofstadter system. It has been shown that the largely anisotropic hopping (the quasi-one-dimension limit) is required for the emergence of butterflylike gaps [10]. The results are confirmed in our numerical calculation under the condition $J_{x} \gg T_{y}, T_{z}$ with the $x$ axis as the conductive axis. In the numerical calculation, we have further assumed the effective magnetic field $\mathbf{B}=\left(0, B_{0} \sin \Theta, B_{0} \cos \Theta\right)$ with an angle $\Theta$ tilted from $z$ axis, which corresponds to the magnetic fluxes $\Phi_{3}=0$ and $\Phi_{1} / \Phi_{2}=\phi_{x} / \varphi_{x}=-\tan \Theta$. In Fig. 5, we plot the energy spectra against the angle $\Theta$ for typical hopping and magnetic strengths. When $T_{y}=T_{z}=0.5 J_{x}$ in Figs. 5(a) (with $B_{0}=1$ ) and 5(b) (with $B_{0}=0.2$ ), the energy spectra in this three-dimensional system does not have many
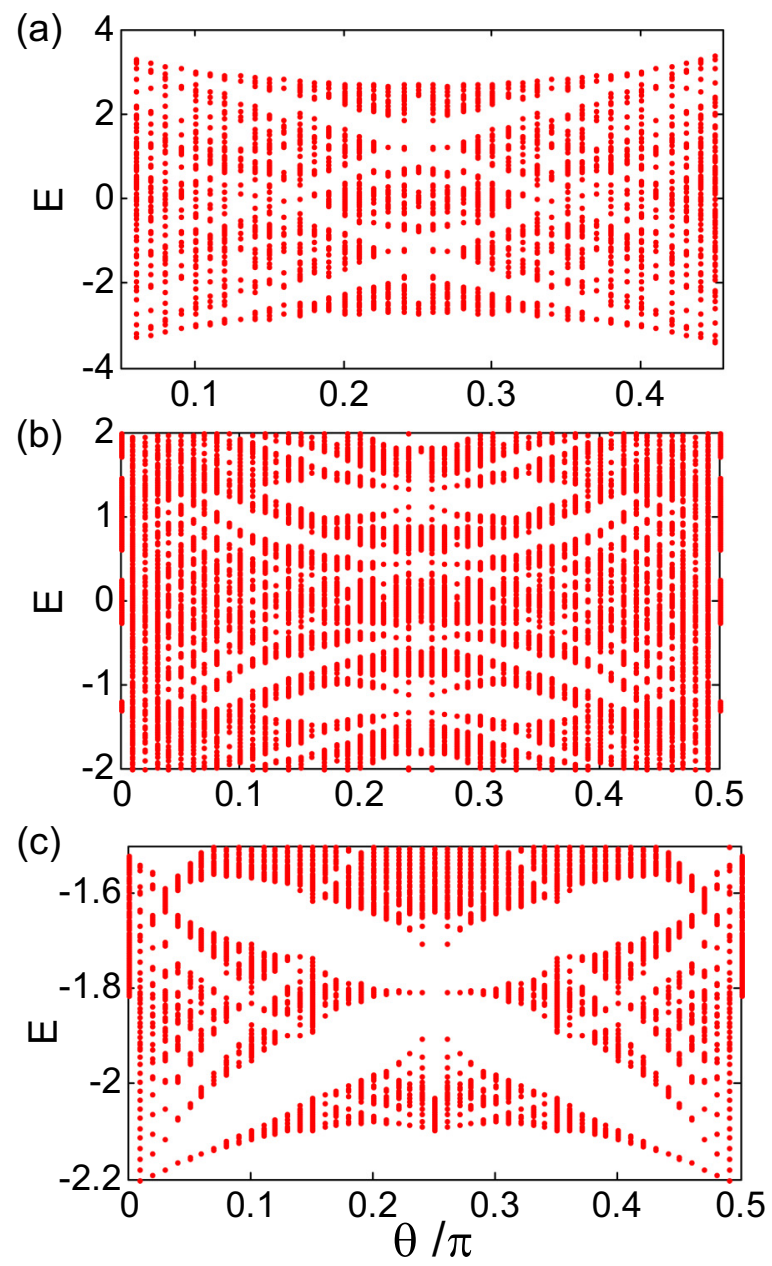

FIG. 5. The energy spectra $E$ as a function of the angle of the effective magnetic field $\Theta$. (a) $T_{y}=T_{z}=0.5$ and $B_{0}=1$; (b) $T_{y}=$ $T_{z}=0.5$ and $B_{0}=0.2$; (c) $T_{y}=T_{z}=0.1$ and $B_{0}=0.2$. The energy unit is set as $J_{x}=1$.

gaps (aside from the trivial Bragg-reflection gaps) with the fractal structure since many of them are wiped out. In the largely anisotropic case of $T_{y}=T_{z}=0.1 J_{x}$ in Fig. 5(c), which corresponds to a quasi-one-dimensional system, a structure akin to the butterfly seen in the bottom (or at the top) of the whole spectrum emerges for certain regions of parameters (with $B_{0}=0.2$ ). In experiments, the energy spectrum of this generalized Hofstadter model may be probed from the density distributions of ultracold fermions in the optical lattice with an external trap [72].

\section{B. The Chern numbers}

It is well known that the integer quantum Hall effect exhibits in the two-dimensional Hofstadter system and is topologically characterized by a Chern number. Previous work has been devoted to the generalization of the integer quantized Hall effect in three-dimensional systems [7-10]. It has been proven that every quantized invariant on a $d$-dimensional torus $T^{d}$ is a function of the $d(d-1) / 2$ sets of Chern numbers obtained by slicing $T^{d}$ by the $d(d-1) / 2$ distinct $T^{2}$ [73]. In our threedimensional Hofstadter system with $d=3$, the topological invariants for the quantized Hall effect are given by three 
Chern numbers $\mathbf{C}=\left(C_{x y}, C_{x z}, C_{y z}\right)$ for three two-dimensional planes. Here we have $C_{y z}=0$ for the trivial $y z$ plane since the magnetic flux $\Phi_{3}$ at this plane is assumed to be zero. Following the approach in Refs. [8,9], when the Fermi energy lies in an energy gap between two bands $N$ and $N+1$ in this system, the other two Chern numbers $C_{x s}$ with $s=y, z$ are given by (let $a=1$ )

$$
C_{x s}=\frac{1}{2 \pi} \sum_{n \leqslant N} \int_{-\pi}^{\pi} d k_{s^{\prime}} c_{x s}^{(n)}\left(k_{s^{\prime}}\right),
$$

where $s^{\prime}$ denotes replacing $s$ between $y$ and $z$, and the Chern number $c_{x s}^{(n)}\left(k_{s^{\prime}}\right)$ for the $n$th filling band (or $n$th occupied Bloch state) is defined on the torus $T^{2}$ spanned by $k_{x}$ and $k_{s}$ :

$$
c_{x s}^{(n)}\left(k_{s^{\prime}}\right)=\frac{1}{2 \pi} \int_{-\pi / \tilde{q}}^{\pi / \tilde{q}} d k_{x} \int_{-\pi}^{\pi} d k_{s} F_{x s}^{(n)}(\mathbf{k}),
$$

where the corresponding Berry curvature $F_{x s}^{(n)}(\mathbf{k})=$ $\operatorname{Im}\left(\left\langle\partial_{k_{s}} u_{n}(\mathbf{k}) \mid \partial_{k_{x}} u_{n}(\mathbf{k})\right\rangle-\left\langle\partial_{k_{x}} u_{n}(\mathbf{k}) \mid \partial_{k_{s}} u_{n}(\mathbf{k})\right\rangle\right)$ is a topological expression as a generalization of the results in two dimensions [3].

The determination of the Chern numbers is a delicate procedure that can be achieved explicitly only in the largely anisotropic cases with a one-to-one correspondence between the Hall conductivities on the three-dimensional and twodimensional Hofstadter systems [8-10]. By generalizing the efficient way based on the U(1) link to obtain the Berry curvature in the discrete Brillouin zone [74], we can numerically calculate the Chern numbers $C_{x s}$ given by Eq. (9) for all the regions of hopping parameters. Note that the Brillouin zone is also discrete in realistic experiments due to the finite lattice. Here the so-called $\mathrm{U}(1)$ link is defined as $U_{\eta}^{(n)}\left(\mathbf{k}_{\mathbf{J}}\right) \equiv$ $\left\langle u_{n}\left(\mathbf{k}_{\mathbf{J}}\right) \mid u_{n}\left(\mathbf{k}_{\mathbf{J}+\hat{\eta}}\right)\right\rangle /\left|\left\langle u_{n}\left(\mathbf{k}_{\mathbf{J}}\right) \mid u_{n}\left(\mathbf{k}_{\mathbf{J}+\hat{\eta}}\right)\right\rangle\right|$ for each pixel $\mathbf{k}_{\mathbf{J}}=$ $\left(k_{x}, k_{y}, k_{z}\right)$ of the discrete three-dimensional Brillouin zone, where $\hat{\eta}=\hat{x}, \hat{s}$ is a unit vector along the axis. From the U(1) link, the manifestly gauge-invariant Berry curvature is given by [74]

$$
\mathcal{F}_{x s}^{(n)}\left(\mathbf{k}_{\mathbf{J}}\right)=i \ln \frac{U_{x}^{(n)}\left(\mathbf{k}_{\mathbf{J}}\right) U_{s}^{(n)}\left(\mathbf{k}_{\mathbf{J}+\hat{x}}\right)}{U_{x}^{(n)}\left(\mathbf{k}_{\mathbf{J}+\hat{s}}\right) U_{s}^{(n)}\left(\mathbf{k}_{\mathbf{J}}\right)},
$$

where $\mathcal{F}_{x s}^{(n)}\left(\mathbf{k}_{\mathbf{J}}\right)$ corresponds to a discrete version of the Berry curvature on the finite lattice. The Chern numbers $C_{x s}$ can be extracted as

$$
C_{x s}=\frac{1}{4 \pi^{2}} \sum_{n \leqslant N} \sum_{\mathbf{J}} \mathcal{F}_{x s}^{(n)}\left(\mathbf{k}_{\mathbf{J}}\right) .
$$

With the above equation, we can numerically calculate the Chern numbers $C_{x s}$ for the three-dimensional quantum Hall effect in the absence of the band crossing.

We first consider the cases in which all the $\tilde{q}$ bands are totally nonoverlapping with $N_{g}=\tilde{q}-1$ gaps, as shown in Figs. 3(a), 3(b), and 4. In these cases, the U(1) field strength $\mathcal{F}_{x s}^{(n)}$ is well defined in the whole three-dimensional momentum space as there is no degeneracy of the Bloch wave functions $\left|u_{n}(\mathbf{k})\right\rangle$, which are numerically obtained by solving the eigenvalue equation (8). In our numerical results, we find that $c_{x s}^{(n)}$ are independent of the momentum $k_{s^{\prime}}$ in these cases and thus one has a simpler expression of the Chern numbers $C_{x s}=\sum_{n \leqslant N} c_{x s}^{(n)}(0)$. In Fig. 4, the corresponding
Chern numbers $C_{x y}$ and $C_{x z}$ when the Fermi level lies in each band gap are plotted. In Figs. 4(a) and 4(b), one can find that there is always one nonzero number $C_{x y}$ or $C_{x z}$ for each of the $N_{g}=4$ gaps. More interestingly, as shown in Figs. 4(c) and 4(d), the three-dimensional Hall system can have two nonzero Chern numbers, such as $C_{x y}=C_{x z}=-1$ and $C_{x y}=C_{x z}=1$ when the Fermi level lies in the third and fourth gaps, respectively.

In the presence of band crossing or touching, such as the cases shown in Figs. 3(c) and 3(d), the U(1) Berry curvature is no longer well defined in the whole momentum space due to certain degeneracies of the Bloch wave function along the $k_{x}$ direction. To overcome this problem in the numerical calculation of the Berry curvature, we introduce a generalized periodic (twisted) boundary condition [75] to Hamiltonian (6) with the wave function of the $n$th occupied Bloch state: $\left|u_{n}\left(m+L_{x}, \alpha, k_{y}, k_{z}\right)\right\rangle=e^{i \alpha}\left|u_{n}\left(m, \alpha, k_{y}, k_{z}\right)\right\rangle$ (here $1 \leqslant m \leqslant$ $L_{x}$ ), where $L_{x}$ denotes the lattice length in the $x$ axis and $\alpha \in[-\pi, \pi]$ is the twist angle. Under this boundary condition, the Chern number $c_{x s}^{(n)}\left(k_{s^{\prime}}\right)$ in Eq. (9) becomes [75]

$$
c_{x s}^{(n)}\left(k_{s^{\prime}}\right)=\frac{1}{2 \pi} \int_{-\pi}^{\pi} d \alpha \int_{-\pi}^{\pi} d k_{s} F_{x s}^{(n)}(\tilde{\mathbf{k}}),
$$

where $\tilde{\mathbf{k}} \equiv\left(\alpha, k_{y}, k_{z}\right)$ is the generalized momentum space. By replacing $k_{x}$ with $\alpha$ [i.e., $\mathbf{k}_{\mathbf{J}} \rightarrow \tilde{\mathbf{k}}_{\mathbf{J}}=\left(\alpha, k_{y}, k_{z}\right)$, we numerically diagonalize the tight-binding Hamiltonian (6) under the twisted boundary condition and obtain the energies $E_{n}(\tilde{\mathbf{k}})$ and the corresponding eigenfunctions $\left|u_{n}(\tilde{\mathbf{k}})\right\rangle$. The results show that the energies $E_{n}(\tilde{\mathbf{k}})$ for different $n$ are no longer overlapped in $\tilde{\mathbf{k}}$ space. Therefore, the same procedure of numerically calculating the Chern numbers $C_{x s}$ based on the U(1)-link method can be implemented by replacing $k_{x}$ with $\alpha$. As shown in Figs. 3(c) and 3(d), the three Chern numbers $\mathbf{C}=\left(C_{x y}, C_{x z}, C_{y z}\right)$ when the Fermi level lies in each energy gap are plotted. The results again demonstrate that the quantum Hall effect in this three-dimensional Hofstadter system is topologically characterized by one or two nonzero Chern numbers. We note that the Chern numbers of the Bloch bands without overlapping in the previous cases can also be numerically obtained in this way under the twisted boundary condition, which has been confirmed in our numerical simulations.

In the artificially generated Hofstadter bands in twodimensional optical lattices [51-53], the Chern number has been experimentally extracted from the center-of-mass drift of an ultracold atomic cloud [53]. This method can be directly used in the three-dimensional Hofstadter system to detect the Chern numbers $C_{x s}$. One can apply a constant force created by an optical gradient along the $s$ direction; the atomic cloud on the lattice will undergo Bloch oscillations along this direction. When the filled bands have nonzero Berry curvature $F_{x s}^{(n)}$, the atomic cloud will experience a net drift along the $x$ axis in response to the force and one can measure its center-of-mass evolution to extract the Chern numbers $C_{x s}$ with high accuracy [53], which is analogous to the quantum-Hall-response measurement. The displacement of the atomic cloud along $x(-x)$ direction gives the corresponding positive (negative) Chern value. Thus in experiments, for the cases of two nonzero Chern numbers, one can observe two significant displacements of the atomic cloud when varying the direction of the applied force 
from the $y$ axis to the $z$ axis, in contrast to a single displacement for the cases of one nonzero Chern number. The applied force can be chosen to be strong enough to accurately detect the displacement, but weak enough to limit the Landau-Zener transitions to higher bands. An alternative method to determine the Chern numbers would be directly measuring the Berry curvature by the newly developed technique of tomography of Bloch bands in optical lattices, as proposed and demonstrated in Refs. [54-57].

We further consider the energy gap $E_{g}$ of the topological nontrivial bands in this system, which is defined by the energy difference between the top of the filled bands and the bottom of the empty bands. In our numerical results, we find that the gaps for filled topological bands with one nonzero Chern number can be as large as $E_{g} \approx 1\left(J_{x}=1\right.$ as the energy unit), such as the filled bands with $C_{x z}= \pm 1$ in Fig. 3(c). Even for the other bands shown in Fig. 3, the corresponding gaps can be up to the hopping strengths $T_{y, z}$. For the filled bands with two nonzero Chern numbers shown as the dashed lines in Figs. 3(b) (also in Fig. 4) and 3(d), the gaps are numerically obtained as $E_{g} \approx 0.18$ and $E_{g} \approx 0.3$, respectively. So they are also comparable to $T_{y, z}=0.4$ or 0.5 , which would be large enough for the measurement of their Chern numbers in realistic experiments [53,56,57]. When the Fermi level lies in the butterflylike gaps shown in Fig. 5(c), there also are one or two nonzero Chern numbers but the gaps there are small due to the small hopping parameters $T_{y}=T_{z}=0.1$. For instance, when the Fermi energy is $E_{F}=-0.19$ and $\Theta=$ $\arctan (3 / 4) \approx 0.2 \pi$, the gap for the filled band is $E_{g} \approx 0.18$, which is one of the primary gaps in the spectrum shown in Fig. 5(c). We numerically calculate the Chern numbers and obtain $\mathbf{C}=(-1,0,0)$ in this case with the fluxes $\Phi_{1}=3 / 25$ and $\Phi_{2}=4 / 25$. When the Fermi energy $E_{F}=-0.19$ and $\Theta=\arctan (5 / 12) \approx 0.126 \pi$, the gap for the filled band in Fig. 5(c) is $E_{g} \approx 0.05$, and the obtained Chern numbers are $\mathbf{C}=(1,-1,0)$ with the corresponding fluxes $\Phi_{1}=5 / 65$ and $\Phi_{2}=12 / 65$ in this case.

Finally we consider the topological transition between the bands of different Chern numbers C. Without loss of generality, we consider the three-dimensional Hofstader system with parameters and bands shown in Figs. 4(c) and 4(d) and the Fermi energy $E_{F}$ initially lies in the third gap with two nonzero Chern numbers $\mathbf{C}=(-1,-1,0)$. In experiments, one can observe two significant displacements of the atomic cloud along the $-x$ direction as a Hall response to the applied force from the $y$ to the $z$ axis. When increasing $E_{F}$ to lie inside the fourth gap, the topological transition results with the Chern numbers become $\mathbf{C}=(1,1,0)$, such that both the two atomic Hall drifts change direction (along the $x$ direction). Increasing $E_{F}$ up to be inside the fifth and sixth gaps, two topological transitions happen sequentially with the Chern numbers becoming $\mathbf{C}=(0,1,0)$ and $\mathbf{C}=(1,0,0)$, respectively, in which cases one of the two drifts disappears. When all the bands are filled with $\mathbf{C}=(0,0,0)$, there will be vanishing displacement as a response to the force of varying directions. For the Fermi energy in the bands, the system is in the conducting phase and the Chern numbers are ill defined. One can also turn the parameters of the effective magnetic fluxes for fixed $E_{F}$ to induce the topological transition. For instance, one can vary the parameter $\Theta$ in Fig. 5(c) from $\pi / 8$ to $3 \pi / 8$ with fixed $E_{F}=-0.19$. In this case, there are four sequent topological transitions with varying $\mathbf{C}=$ $(1,-1,0) \rightarrow(-1,0,0) \rightarrow(0,-1,0) \rightarrow(-1,1,0)$ for the four primary gaps, which can similarly be revealed from different atomic displacements in the Hall-response measurements.

\section{CONCLUSION}

In summary, we have proposed an experimental scheme to realize a tunable generalized three-dimensional Hofstadter Hamiltonian with ultracold atoms in a cubic optical lattice, which describes a lattice system under effective magnetic fluxes in three dimensions. We have shown that the Weyl points and nodal loops can respectively emerge in the bulk bands of this system for certain hopping configurations. Moreover, we have illustrated that with proper rational fluxes and hopping parameters, the system can exhibit the three-dimensional quantum Hall effect when the Fermi level lies in the band gaps, which is topologically characterized by one or two nonzero Chern numbers. Our proposed optical-lattice system provides a powerful platform for exploring exotic topological semimetals and insulators in three dimensions that are rare in solid-state materials.

\section{ACKNOWLEDGMENTS}

This work was supported by the NSFC (Grants No. 11604103 and No. 11474153), the NKRDP of China (Grant No. 2016YFA0301803), the NSF of Guangdong Province (Grant No. 2016A030313436), and the Startup Foundation of SCNU.
[1] K. V. Klitzing, G. Dorda, and M. Pepper, New Method for High-Accuracy Determination of the Fine-Structure Constant Based on Quantized Hall Resistance, Phys. Rev. Lett. 45, 494 (1980).

[2] D. C. Tsui, H. L. Stormer, and A. C. Gossard, Two-Dimensional Magnetotransport in the Extreme Quantum Limit, Phys. Rev. Lett. 48, 1559 (1982).

[3] D. J. Thouless, M. Kohmoto, M. P. Nightingale, and M. den Nijs, Quantized Hall Conductance in a Two-Dimensional Periodic Potential, Phys. Rev. Lett. 49, 405 (1982).
[4] R. B. Laughlin, Anomalous Quantum Hall Effect: An Incompressible Quantum Fluid with Fractionally Charged Excitations, Phys. Rev. Lett. 50, 1395 (1983).

[5] F. D. M. Haldane, Model for a Quantum Hall Effect without Landau Levels: Condensed-Matter Realization of the "Parity Anomaly", Phys. Rev. Lett. 61, 2015 (1988).

[6] D. R. Hofstadter, Energy levels and wave functions of Bloch electrons in rational and irrational magnetic fields, Phys. Rev. B 14, 2239 (1976). 
[7] B. I. Halperin, Possible states for a three-dimensional electron gas in a strong magnetic field, Jpn. J. Appl. Phys. 26, 1913 (1987).

[8] G. Montambaux and M. Kohmoto, Quantized Hall effect in three dimensions, Phys. Rev. B 41, 11417 (1990).

[9] M. Kohmoto, B. I. Halperin, and Y.-S. Wu, Diophantine equation for the three-dimensional quantum Hall effect, Phys. Rev. B 45, 13488 (1992).

[10] M. Koshino, H. Aoki, K. Kuroki, S. Kagoshima, and T. Osada, Hofstadter Butterfly and Integer Quantum Hall Effect in Three Dimensions, Phys. Rev. Lett. 86, 1062 (2001).

[11] M. Z. Hasan and C. L. Kane, Colloquium: Topological insulators, Rev. Mod. Phys. 82, 3045 (2010).

[12] X.-L. Qi and S. C. Zhang, Topological insulators and superconductors, Rev. Mod. Phys. 83, 1057 (2011).

[13] X. Wan, A. M. Turner, A. Vishwannath, and S. Y. Savrasov, Topological semimetal and Fermi-arc surface states in the electronic structure of pyrochlore iridates, Phys. Rev. B 83, 205101 (2011).

[14] A. A. Burkov, M. D. Hook, and L. Balents, Topological nodal semimetals, Phys. Rev. B 84, 235126 (2011).

[15] S.-Y. Xu, I. Belopolski, N. Alidoust, M. Neupane, C. Zhang, R. Sankar, S.-M. Huang, C.-C. Lee, G. Chang, B. Wang, G. Bian, H. Zheng, D. S. Sanchez, F. Chou, H. Lin, S. Jia, and M. Z. Hasan, Discovery of a Weyl fermion semimetal and topological Fermi arcs, Science 349, 613 (2015).

[16] B. Q. Lv, H. M. Weng, B. B. Fu, X. P. Wang, H. Miao, J. Ma, P. Richard, X. C. Huang, L. X. Zhao, G. F. Chen, Z. Fang, X. Dai, T. Qian, and H. Ding, Experimental Discovery of Weyl Semimetal TaAs, Phys. Rev. X 5, 031013 (2015).

[17] G. Bian, T.-R. Chang, R. Sankar, S.-Y. Xu, H. Zheng, T. Neupert, C.-K. Chiu, S.-M. Huang, G. Chang, I. Belopolski, D. S. Sanchez, M. Neupane, N. Alidoust, C. Liu, B. Wang, C.-C. Lee, H.-T. Jeng, A. Bansil, F. Chou, H. Lin, and M. Z. Hasan, Topological nodal-line fermions in the non-centrosymmetric superconductor compound $\mathrm{PbTaSe}_{2}$, Nat. Commun. 7, 10556 (2016).

[18] L. Lu, Z. Wang, D. Ye, L. Ran, L. Fu, J. D. Joannopoulos, and M. Soljačić, Experimental observation of Weyl points, Science 349, 622 (2015).

[19] M. Xiao, W.-J. Chen, W.-Y. He, and C.-T. Chan, Synthetic gauge flux and Weyl points in acoustic systems, Nat. Phys. 11, 920 (2015).

[20] L. Balicas, G. Kriza, and F. I. B. Williams, Sign Reversal of the Quantum Hall Number in (TMTSF) ${ }_{2} \mathrm{PF}_{6}$, Phys. Rev. Lett. 75, 2000 (1995).

[21] S. K. McKernan, S. T. Hannahs, U. M. Scheven, G. M. Danner, and P. M. Chaikin, Competing Instabilities and the High Field Phases of (TMTSF) ${ }_{2} \mathrm{ClO}_{4}$, Phys. Rev. Lett. 75, 1630 (1995).

[22] B. A. Bernevig, T. L. Hughes, S. Raghu, and D. P. Arovas, Theory of the Three-Dimensional Quantum Hall Effect in Graphite, Phys. Rev. Lett. 99, 146804 (2007).

[23] K. Mullen, B. Uchoa, and D. T. Glatzhofer, Line of Dirac Nodes in Hyperhoneycomb Lattices, Phys. Rev. Lett. 115, 026403 (2015).

[24] M. Lewenstein, A. Sanpera, V. Ahufinger, B. Damski, A. Sen(De), and U. Sen, Ultracold atomic gases in optical lattices: Mimicking condensed matter physics and beyond, Adv. Phys. 56, 243 (2007).
[25] D.-W. Zhang, Z. D. Wang, and S.-L. Zhu, Relativistic quantum effects of Dirac particles simulated by ultracold atoms, Front. Phys. 7, 31 (2012).

[26] Y.-J. Lin, R. L. Compton, K. Jiménez-García, J. V. Porto, and I. B. Spielman, Synthetic magnetic fields for ultracold neutral atoms, Nature (London) 462, 628 (2009).

[27] Y.-J. Lin, K. Jiménez-García, and I. B. Spielman, A spin-orbit coupled Bose-Einstein condensate, Nature (London) 471, 83 (2011).

[28] P. Wang, Z.-Q. Yu, Z. Fu, J. Miao, L. Huang, S. Chai, H. Zhai, and J. Zhang, Spin-Orbit Coupled Degenerate Fermi Gases, Phys. Rev. Lett. 109, 095301 (2012).

[29] L. W. Cheuk, A. T. Sommer, Z. Hadzibabic, T. Yefsah, W. S. Bakr, and M. W. Zwierlein, Spin-Injection Spectroscopy of a Spin-Orbit Coupled Fermi Gas, Phys. Rev. Lett. 109, 095302 (2012).

[30] Z. Wu, L. Zhang, W. Sun, X.-T. Xu, B.-Z. Wang, S.-C. Ji, Y. Deng, S. Chen, X.-J. Liu, and J.-W. Pan, Realization of twodimensional spin-orbit coupling for Bose-Einstein condensates, Science 354, 83 (2016).

[31] J. Dalibard, F. Gerbier, G. Juzeliūnas, and P. Öhberg, Colloquium: Artificial gauge potentials for neutral atoms, Rev. Mod. Phys. 83, 1523 (2011).

[32] V. Galitski and I. B. Spielman, Spin-orbit coupling in quantum gases, Nature (London) 494, 49 (2013).

[33] N. Goldman, G. Juzeliūnas, P. Öhberg, and I. B. Spielman, Light-induced gauge fields for ultracold atoms, Rep. Prog. Phys. 77, 126401 (2014).

[34] H. Zhai, Degenerate quantum gases with spin-orbit coupling: A review, Rep. Prog. Phys. 78, 026001 (2015).

[35] S. L. Zhu, H. Fu, C. J. Wu, S. C. Zhang, and L. M. Duan, Spin Hall Effects for Cold Atoms in a Light-Induced Gauge Potential, Phys. Rev. Lett. 97, 240401 (2006).

[36] R. O. Umucalilar, H. Zhai, and M. O. Oktel, Trapped Fermi Gases in Rotating Optical Lattices: Realization and Detection of the Topological Hofstadter Insulator, Phys. Rev. Lett. 100, 070402 (2008).

[37] L.-B. Shao, S.-L. Zhu, L. Sheng, D. Y. Xing, and Z. D. Wang, Realizing and Detecting the Quantum Hall Effect without Landau Levels by Using Ultracold Atoms, Phys. Rev. Lett. 101, 246810 (2008).

[38] N. Goldman, I. Satija, P. Nikolic, A. Bermudez, M. A. MartinDelgado, M. Lewenstein, and I. B. Spielman, Realistic TimeReversal Invariant Topological Insulators with Neutral Atoms, Phys. Rev. Lett. 105, 255302 (2010).

[39] A. Bermudez, L. Mazza, M. Rizzi, N. Goldman, M. Lewenstein, and M. A. Martin-Delgado, Wilson Fermions and Axion Electrodynamics in Optical Lattices, Phys. Rev. Lett. 105, 190404 (2010).

[40] B. Béri and N. R. Copper, $\mathbb{Z}_{2}$ Topological Insulators in Ultracold Atomic Gases, Phys. Rev. Lett. 107, 145301 (2011).

[41] S.-L. Zhu, L.-B. Shao, Z. D. Wang, and L.-M. Duan, Probing Non-Abelian Statistics of Majorana Fermions in Ultracold Atomic Superfluid, Phys. Rev. Lett. 106, 100404 (2011).

[42] K. Sun, W. V. Liu, A. Hemmerich, and S. Das Sarma, Topological semimetal in a fermionic optical lattice, Nat. Phys 8, 67 (2012).

[43] L.-J. Lang, X. Cai, and S. Chen, Edge States and Topological Phases in One-Dimensional Optical Superlattices, Phys. Rev. Lett. 108, 220401 (2012). 
[44] S.-L. Zhu, Z.-D. Wang, Y.-H. Chan, and L.-M. Duan, Topological Bose-Mott Insulators in a One-Dimensional Optical Superlattice, Phys. Rev. Lett. 110, 075303 (2013).

[45] D.-L. Deng, S.-T. Wang, and L.-M. Duan, Direct probe of topological order for cold atoms, Phys. Rev. A 90, 041601(R) (2014).

[46] S.-T. Wang, D.-L. Deng, and L.-M. Duan, Probe of ThreeDimensional Chiral Topological Insulators in an Optical Lattice, Phys. Rev. Lett. 113, 033002 (2014).

[47] X.-J. Liu, K. T. Law, and T. K. Ng, Realization of 2D SpinOrbit Interaction and Exotic Topological Orders in Cold Atoms, Phys. Rev. Lett. 112, 086401 (2014).

[48] M. Atala, M. Aidelsburger, J. T. Barreiro, D. Abanin, T. Kitagawa, E. Demler, and I. Bloch, Direct measurement of the Zak phase in topological Bloch bands, Nat. Phys. 9, 795 (2013).

[49] G. Jotzu, M. Messer, R. Desbuquois, M. Lebrat, T. Uehlinger, D. Greif, and T. Esslinger, Experimental realisation of the topological Haldane model with ultracold fermions, Nature (London) 515, 237 (2014).

[50] M. Aidelsburger, M. Atala, S. Nascimbène, S. Trotzky, Y.-A. Chen, and I. Bloch, Experimental Realization of Strong Effective Magnetic Fields in an Optical Lattice, Phys. Rev. Lett. 107, 255301 (2011).

[51] H. Miyake, G. A. Siviloglou, C. J. Kennedy, W. C. Burton, and W. Ketterle, Realizing the Harper Hamiltonian with LaserAssisted Tunneling in Optical Lattices, Phys. Rev. Lett. 111, 185302 (2013).

[52] M. Aidelsburger, M. Atala, M. Lohse, J. T. Barreiro, B. Paredes, and I. Bloch, Realization of the Hofstadter Hamiltonian with Ultracold Atoms in Optical Lattices, Phys. Rev. Lett. 111, 185301 (2013).

[53] M. Aidelsburger, M. Lohse, C. Schweizer, M. Atala, J. T. Barreiro, S. Nascimbène, N. R. Cooper, I. Bloch, and N. Goldman, Measuring the Chern number of Hofstadter bands with ultracold bosonic atoms, Nat. Phys. 11, 162 (2015).

[54] E. Alba, X. Fernandez-Gonzalvo, J. Mur-Petit, J. K. Pachos, and J. J. Garcia-Ripoll, Seeing Topological Order in Time-of-Flight Measurements, Phys. Rev. Lett. 107, 235301 (2011).

[55] P. Hauke, M. Lewenstein, and A. Eckardt, Tomography of Band Insulators from Quench Dynamics, Phys. Rev. Lett. 113, 045303 (2014).

[56] N. Fläschner, B. S. Rem, M. Tarnowski, D. Vogel, D. S. Lühmann, K. Sengstock, and C. Weitenberg, Experimental reconstruction of the Berry curvature in a Floquet Bloch band, Science 352, 1091 (2016).

[57] T. Li, L. Duca, M. Reitter, F. Grusdt, E. Demler, M. Endres, M. Schleier-Smith, I. Bloch, and U. Schneider, Bloch state tomography using Wilson lines, Science 352, 1094 (2016).
[58] J. H. Jiang, Tunable topological Weyl semimetal from simplecubic lattices with staggered fluxes, Phys. Rev. A 85, 033640 (2012).

[59] T. Dubček, C. J. Kennedy, L. Lu, W. Ketterle, M. Soljacic and H. Buljan, Weyl Points in Three-Dimensional Optical Lattices: Synthetic Magnetic Monopoles in Momentum Space, Phys. Rev. Lett. 114, 225301 (2015).

[60] D.-W. Zhang, S.-L Zhu, and Z. D. Wang, Simulating and exploring Weyl semimetal physics with cold atoms in a twodimensional optical lattice, Phys. Rev. A 92, 013632 (2015).

[61] W.-Y. He, S. Zhang, and K. T. Law, Realization and detection of Weyl semimetals and the chiral anomaly in cold atomic systems, Phys. Rev. A 94, 013606 (2016).

[62] Y. Xu and L.-M. Duan, Type-II Weyl points in three-dimensional cold-atom optical lattices, Phys. Rev. A 94, 053619 (2016).

[63] D.-W. Zhang, Y. X. Zhao, R.-B. Liu, Z.-Y. Xue, S.-L. Zhu, and Z. D. Wang, Quantum simulation of exotic $\mathcal{P} \mathcal{T}$-invariant topological nodal loop bands with ultracold atoms in an optical lattice, Phys. Rev. A 93, 043617 (2016).

[64] Y. Xu and C. Zhang, Dirac and Weyl rings in three-dimensional cold-atom optical lattices, Phys. Rev. A 93, 063606 (2016).

[65] K. Shastri, Z. Yang, and B. Zhang, Realizing type-II Weyl points in an optical lattice, Phys. Rev. B 95, 014306 (2017).

[66] D. Jaksch and P. Zoller, Creation of effective magnetic fields in optical lattices: The Hofstadter butterfly for cold neutral atoms, New J. Phys. 5, 56 (2003).

[67] A. R. Kolovsky, Creating artificial magnetic fields for cold atoms by photon-assisted tunneling, Europhys. Lett. 93, 20003 (2011).

[68] In typical cold-atom experiments, the frequencies of the Raman laser $\omega_{j}$ are on the order of $\mathrm{GHz}$, and the natual hopping rate $J_{s} / \hbar$ is less than about $1 \mathrm{kHz}$.

[69] L. Tarruell, D. Greif, T. Uehlinger, G. Jotzu, and T. Esslinger, Creating, moving and merging Dirac points with a Fermi gas in a tunable honeycomb lattice, Nature (London) 483, 302 (2012).

[70] L.-K. Lim, J.-N. Fuchs, and G. Montambaux, Bloch-Zener Oscillations Across a Merging Transition of Dirac Points, Phys. Rev. Lett. 108, 175303 (2012).

[71] P. G. Harper, Single band motion of conduction electrons in a uniform magnetic field, Proc. Phys. Soc. London, Sect. A 68, 874 (1955).

[72] L. Wang and M. Troyer, Seeing Hofstadter's butterfly in atomic Fermi gases, Phys. Rev. A 89, 011603(R) (2014).

[73] J. Avron, R. Seiler, and B. Simon, Homotoyy and Quantization in Condensed Matter Physics, Phys. Rev. Lett. 51, 51 (1983).

[74] T. Fukui, Y. Hatsugai, and H. Suzuki, Chern numbers in discretized Brillouin zone: Efficient method of computing (spin) Hall conductances, J. Phys. Soc. Jpn. 74, 1674 (2005).

[75] Q. Niu, D. J. Thouless, and Y. S. Wu, Quantized Hall conductance as a topological invariant, Phys. Rev. B 31, 3372 (1985). 